\section{Valsalva Retinopathy Associated with Choking and Heimlich Maneuver}

\begin{abstract}
Valsalva is "a particular form of retinopathy, pre-retinal and hemomhagic in nature, secondary to a sudden increase in intrathoracic pressure". Increasing intrathoracic pressure against a closed glottis diminishes venous retum to the heart, decreasing stroke volume and subsequently increasing the venous system pressure. Here we report a case of 52-year-old male who presented with a sudden central vision loss because of macular hemomage. The case occurred following the suffocation, which is not often seen; hence we conducted investigative tests to detemine the cause of retinal bleeding.
\end{abstract}

\section{Case Report}

A 52-year-old Chinese man complained a "black spot" in the vision of his left eye. There was no previous ophthalmic history. $\mathrm{He}$ was under investigation for hypertension.

The man inhaled food three days before seeing the doctor, causing choking, which prompted his wife to beat his chest like doing "Heimlich maneuver". His unaided visual acuity was 1.0 and 1.0 in both eyes. Anterior segment examination was unremarkable. Pupil reactions were normal. Fundus examination of the right eye was normal whereas examination of the left eye revealed a subretinal hemorrhage involving the posterior pole of the left eye (Figure 1). Fundus fluorescein angiography (FFA) showed hypofluorescence in the macular region, due to blockage by hemorrhage. The total size of the lesion was about six disc areas; no large area of fluorescein leakage was found (Figure 2).

One week later his left visual acuity suddenly dropped to 0.05 . We found macular and vitreous hemorrhage during fundus examination. Two months later, indocyanine green angiography (ICGA) showed that vitreous hemorrhage, partial low fluorescence, no fluorescence leakage. After six months, his best-corrected visual acuity recovered to 1.0 , examination indicated that in the left eye the vitreous was clear, and there were pigmentary disorders in macular area. Fifteen months later, the corrected visual acuity of the left eye remained at 1.0 with some pigmentary changes in the macular area.

\section{Discussion}

Vitreous hemorrhage is one of the most common causes of sudden, painless loss of vision. We have reviewed the related case reports from 2002 to 2012 in PubMed, and summarized reported literature about vitreous hemorrhage and macula hemorrhage in the Table 1.

Valsalva retinopathy is a unilateral or bilateral condition that occurs when increased intra-thoracic or intra-abdominal pressure transmitted to the eye causes a sharp rise in the intraocular venous pressure and rupture of superficial retinal capillaries [1,2]. Patients usually give a history of sudden visual loss following a bout of heaving lifting, coughing or vomiting [3]. These hemorrhagic lesions are typically self-limiting and resolve without any complications. There

\section{Journal of Ocular Biology}

Ying-Xiang Huang*, Na Li and Yan-ling Wang

Department of Ophthalmology, Beijing Friendship Hospital, Capital Medical University, Beijing, China

*Address for Correspondence

Ying-Xiang Huang, MD, Chief Ophthalmologist and Associate Professor, Department of Ophthalmology, Beijing Friendship Hospital, Capital Medical University, Beijing, China, E-mail: bshuang@163.com

Submission: 02 August 2013

Accepted: 11 September 2013

Published: 16 September 2013

Reviewed \& Approved by: Eric M Shrier

Department of Ophthalmology

SUNY-Downstate Medical Center, USA is no evidence of increased risk of recurrence. A diagnosis of Valsalva retinopathy is only made after exclusion of other causes of retinal hemorrhages [1,2].

Valsalva retinopathy classically presents with pre-retinal/ subhyaloid hemorrhages and in this case, it's subretinal. Subretinal hemorrhage refers to blood located between the neurosensory retina and the retinal pigment epithelium (RPE). Blood in this space can arise from the choroidal and/or the retinal circulation.

Subretinal hemorrhage may arise simply from choriocapillary hemorrhage through discontinuities in Bruch's membrane. Most of them may arise directly from choroidal neovascular membranes or from a break in the Bruch's membrane/RPE complex. In the case, we have not found any evidence of choroidal neovascularization (CNV) and choroidal rupture. As we know, the outer capillary network runs from the inner plexiform layer to the outer plexiform layer thought the inner nuclear layer [4]. The inner capillaries lie in the ganglion

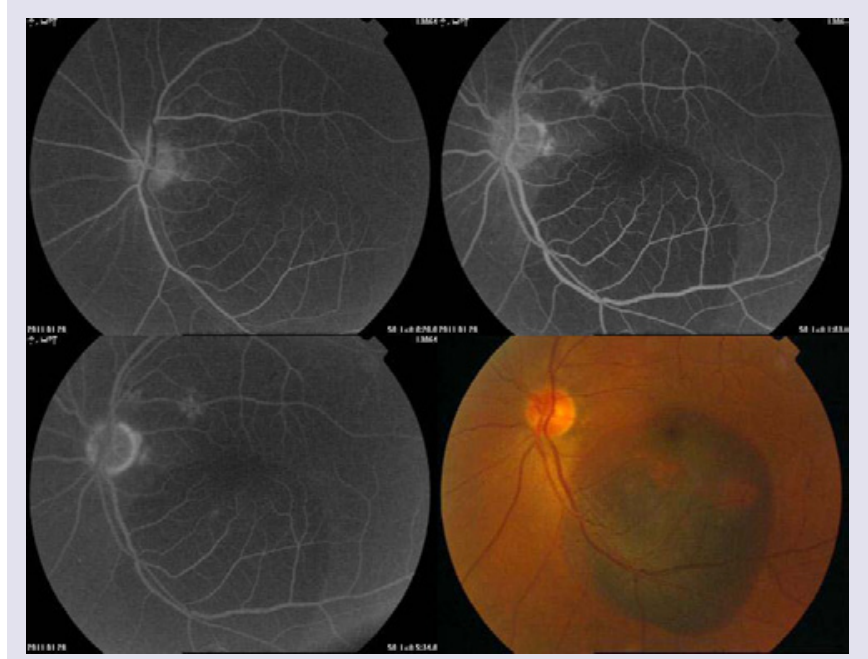

Figure 1: The left eye showed a partial subretinal hemorrhage in the posterior pole and macula one day after asphyxia and no fluorescence leakage. UPPER LEFT: The early phase of FFA; UPPER RIGHT: subretinal hemorrhage blocked fluorescence; LOWER LEFT: the later phase of FFA: no leakage. LOWER RIGHT: color image. 
cell layers under and parallel to the RPCs [4]. Thus, any retinal vascular abnormality or retinal trauma that causes retinal endothelial compromise (inner blood-retinal barrier) will predispose to formation of subretinal hemorrhage. The retinal vessels bleed, with resultant dissection into the subretinal space, forming subretinal hemorrhage and, at times, intraretinal hemorrhage, vitrous hemorrhage and choroidal hemorrhage. Asphyxia or chest compressions may increase the circulation pressure. After asphyxia, the intrathoracic pressure increases against a closed glottis. This diminishes venous return to the heart, decreasing stroke volume and subsequently increasing the venous system pressure $[5,6]$. A sudden rise in intraocular venous pressure causes retinal capillaries to spontaneously rupture.

In this case, there was no typical manifestation of reddish-orange, spheroidal, polyp-like structures or polypoidal vascular lesions at the

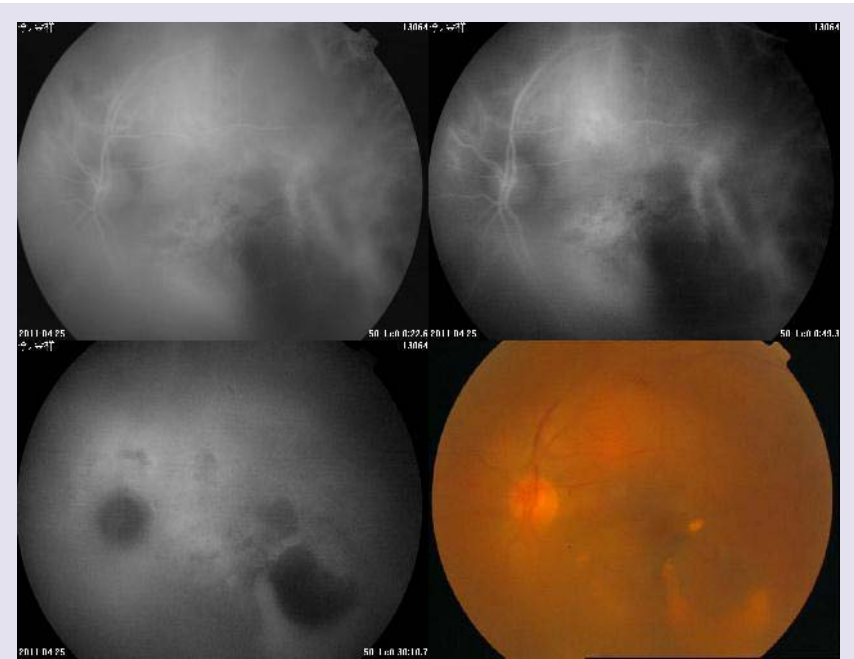

Figure 2: Indocyanine green angiogram showed macular hemorrhage (and mild vitreous hemorrhage) three months after asphyxia. UPPER LEFT: The early phase of ICG; UPPER RIGHT: No polyp-like structures or polypoidal vascular lesions at the termination of a network of large choroidal vessels in the patient's fundus photograph; LOWER LEFT: the later phase of ICG showed only hemorrhage block. LOWER RIGHT: color image.

Table 1: The reported literatures about vitreous hemorrhage and macula hemorrhage from 2002 to 2012 in PubMed.

\begin{tabular}{|c|c|}
\hline & Reason (Number) \\
\hline $\begin{array}{l}\text { Original } \\
\text { research }\end{array}$ & $\begin{array}{l}\text { Age-related macular degeneration (16), Polypoidal choroidal } \\
\text { vasculopathy (9), Proliferative diabetic retinopathy (8), Retinal } \\
\text { vasculitis and branch retinal vein occlusion (8), Terson } \\
\text { syndrome (7), Eales disease (5), Thrombocytopenic purpura } \\
\text { (3), Valsalva retinopathy (3) }\end{array}$ \\
\hline Case report & $\begin{array}{l}\text { Uveal melanoma, Systemic anticoagulants, Choroidal } \\
\text { melanoma, Retinal detachment, Postoperative } \\
\text { endophthalmitisis, Multiple sclerosis-associated uveitis, } \\
\text { (2,respectively) } \\
\text { Persistent hyaloid artery, Proliferative sickle cell retinopathy, } \\
\text { whole-body vibration training, Retinal vasculitis associated } \\
\text { with mixed connective tissue disease, Retinal astrocytic } \\
\text { hamartoma, Vitamin K deficiency bleeding, Lung cancer } \\
\text { from choroidal metastasis, Antiphospholipid syndrome, } \\
\text { Unilateral frosted branch angiiti, Leber congenital amaurosis, } \\
\text { Wegener granulomatosis, Aplastic anemia, retinal artery } \\
\text { macroaneurysms, Autosomal dominant syndrome of retinal } \\
\text { arterial tortuosity, Acquired factor VIII inhibitor, Acute } \\
\text { posterior vitreous detachment, Subarachnoid hemorrhage, } \\
\text { Retinoblastoma Retinoblastoma, (1. respectively) }\end{array}$ \\
\hline
\end{tabular}

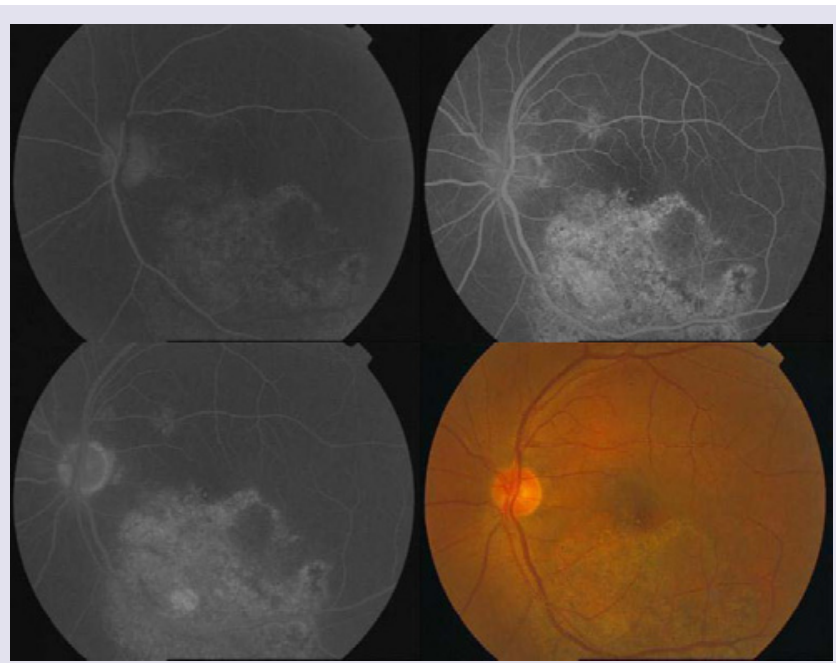

Figure 3: The left eye in FFA fifteen months later showed some pigmentary disorder below the macula. UPPER LEFT: The early phase of FFA; UPPER RIGHT: The venous phase; LOWER LEFT: the later phase of FFA: no leakage. LOWER RIGHT: color image.

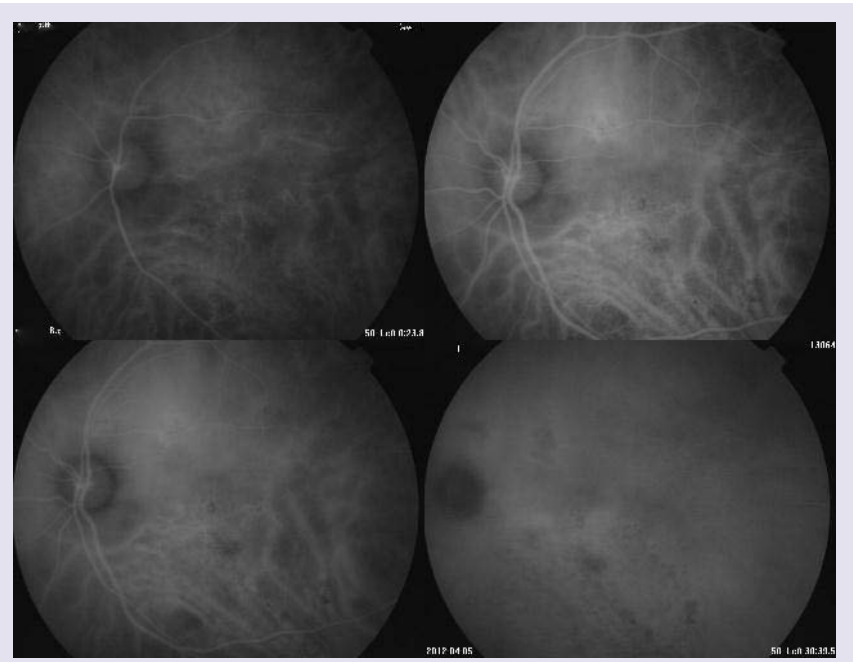

Figure 4: Indocyanine green angiogram showed that the inferior macula was surrounded by chaotic retinal pigment epithelia fifteen months after asphyxia. UPPER LEFT: The early phase of ICG 23 seconds; UPPER RIGHT: 6 minutes later; LOWER LEFT: 10 minutes later. LOWER RIGHT: 30 minutes later.

termination of a network of large choroidal vessels in the patient's fundus photograph. We did not discover any evidences of PCV in ICGA. Also, no characteristics of AMD or pathological changes of neovascularization and effusion appeared. We reckoned that since the patient was overweight and had a history of hypertension, during the process of rescue, the superior vena cava pressure suddenly lifted, induced the reflux of retinal vein blood, and caused acute deep subretinal hemorrhage.

The data suggested Valsalva retinopathy with sub-hyaloid haemorrhage, probably secondary to coughing on a background of hypertension. This report highlights the need to perform a basic systemic examination in similar cases, as incidental findings requiring systemic treatment may be detected. There is no evidence 


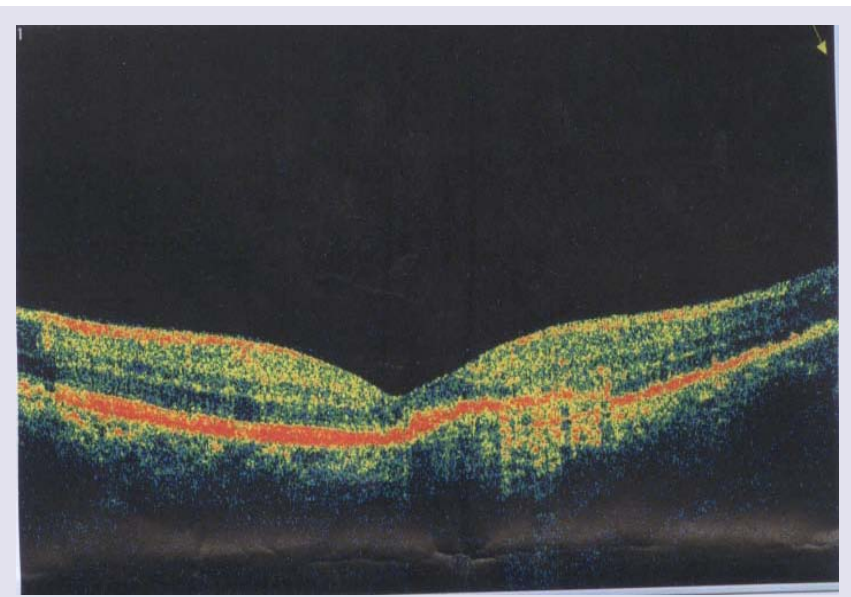

Figure 5: OCT indicated the disordered retinal pigment epithelial layer fifteen months after asphyxia.

of increased risk of recurrence [7]. In the relevant reports about Valsalva retinopathy, it happened in rare condition [2,8]: A 36-yearold Caucasian woman had Valsalva retinopathy after vomiting during pregnancy [8]; A 52-year-old Caucasian man had premacular bleeding due to vomiting after general anaesthesia [2]; A 24-year-old Caucasian man had a macular hemorrhage after thoracic trauma [2]; some men developed a premacular bleed after vigorous dancing [2,9]; some persons developed a premacular hemorrhage after weightlifting [2,3]. A 17-year-old man presented in his recent oratorical contest [10]. There are many case reports of Valsalva. The reasons are different such as choking asthma attacks, motorcycle riding, colonscopy and others [11-18]. A Diagnosis of Valsalva retinopathy is only made after exclusion of other causes of retinal hemorrhages [1,2].

In summary, several reasons will lead to vitreous hemorrhage or macular hemorrhage and it is important to find out the reason. ICGA is a useful tool for the identification and differentiation of Valsalva, PCV and AMD. And we should ask for the patients' history carefully. It is very useful for the diagnosis.

\section{References}

1. Al-Mujaini AS, Montana CC (2008) Valsalva retinopathy in pregnancy: a case report. J Med Case Rep 2: 101.

2. García Fernández M, Navarro JC, Castaño CG (2012) Long-term evolution of Valsalva retinopathy: a case series. J Med Case Rep 6: 346.

3. Chapman-Davies A, Lazarevic A (2002) Valsalva maculopathy. Clin Exp Optom 85: $42-45$

4. Zhang HR (1994) Scanning electron-microscopic study of corrosion casts on retinal and choroidal angioarchitecture in man and animals. Prog Ret Eye Res 13: 243-270.

5. Choi SW, Lee SJ, Rah SH (2006) Valsalva retinopathy associated with fiberoptic gastroenteroscopy. Can J Ophthalmol 41: 491-493.

6. Saricaoglu MS, Kalayci D, Guven D, Karakurt A, Hasiripi H (2009) Decompression retinopathy and possible risk factors. Acta Ophthalmol 87: 94-95.

7. Kashani S, Aslam SA, Bessant D (2009) Valsalva retinopathy presenting with subhyaloid haemorrhage. Emerg Med J 26: 74.

8. Ramskold LA, Asaria RH (2012) Valsalva retinopathy secondary to hyperemesis gravidarum. Eur J Obstet Gynecol Reprod Biol 162: 118-119.

9. Bar-Sela SM, Moisseiev J (2007) Valsalva retinopathy associated with vigorous dancing in a discotheque. Ophthalmic Surg Lasers Imaging 38: 6971.

10. Kim JY, Lee DH, Lee JH, Yoon leN (2009) Valsalva retinopathy associated with an oratorical contest. Korean J Ophthalmol 23: 318-320.

11. Lee VY, Liu DT, Chan WM, Lam DS (2005) Valsalva retinopathy as a complication of colonoscopy. J Clin Gastroenterol 39: 643.

12. Williams MA, Gawley SD, Sharkey JA, McGinnity GF (2012) Cartwheel retinopathy. Clin Exp Optom 95: 244-245.

13. Ho LY, Abdelghani WM (2007) Valsalva retinopathy associated with the choking game. Semin Ophthlmol 22: 63-65.

14. Ioannidis AS, Tranos PG, Harris M (2004) Valsalva retinopathy associated with riding a motorcycle. Eye (Lond) 18: 329-331.

15. Gen H, Kei S, Minoru O, Yutaka I, Soiti CM, et al. (2013) Valsalva Retinopathy Developing During Japanese Cheerleading Training (“Ouendan"). Retin Cases Brief Rep 7: 78-81.

16. Lavezzo MM, Zacharias LC, Takahashi WY (2012) Sub-internal limiting membrane hemorrhage in Valsalva retinopathy: case report. Arq Bras Oftalmol 75: 436-438.

17. Oboh AM, Weilke F, Sheindlin J (2004) Valsalva retinopathy as a complication of colonoscopy. J Clin Gastroenterol 38: 793-794.

18. Rauen M, Oetting TA, Weingeist TA (2007) Valsalva Retinopathy: Vision loss after asthma attack. Eye Rounds.org. 